\title{
Injectable Rectifiers as Microdevices for Remote Electrical Stimulation: an Alternative to Inductive Coupling
}

\author{
A. Ivorra ${ }^{1}$, J. Sacristán ${ }^{2}$ and A. Baldi ${ }^{2}$ \\ ${ }^{1}$ Department of Information and Communication Technologies, Universitat Pompeu Fabra, Barcelona, Spain \\ ${ }^{2}$ Centro Nacional de Microelectrónica (CNM-IMB), CSIC, Campus UAB, Cerdanyola del Vallès, Spain
}

\begin{abstract}
Miniaturization of implantable medical electronic devices is currently compromised by the available means for electrically powering them. Most common energy supply techniques for implants - batteries and inductive couplers - comprise bulky parts which, in most cases, are significantly larger than the circuitry they feed. For overcoming such miniaturization bottleneck in the case of implants for electrical stimulation, we recently proposed and demonstrated a method based on making the implants operate as rectifiers of bursts of high frequency current supplied by remote electrodes. In this way, low frequency current is generated locally around the implant and this low frequency current performs stimulation of excitable tissues whereas the high frequency current only causes innocuous heating. The present paper reports further progress in this technology. We first describe construction and functional test of an injectable stimulator consisting of a single miniature diode $(300 \mu \mathrm{m} \times 300 \mu \mathrm{m} \times 600 \mu \mathrm{m})$ and two thin electrodes which is implanted trough a $19 \mathrm{G}$ needle into an anesthetized earthworm. We then propose a circuit architecture for implementing smart implants based on this technology. Both accomplishments are steps towards the implementation of injectable addressable microsystems for neuroprosthetics. These systems based on the proposed technology will look like short pieces of flexible thread rather than rigid capsules, as it was the case of previous miniature electrical stimulation implants. With currently available microelectronic techniques, very thin stimulation implants (diameter < $200 \mu \mathrm{m}$ ) are easily conceivable. This technology may be foundational to a broad range of new developments in the field of implantable medical devices with applications ranging from wound healing to nerve stimulation for pain relief. In addition, other non-medical devices could also emerge such as implantable identification devices.
\end{abstract}

Keywords - Rectifiers, Microsystems, Implantable Devices, Electrical Stimulation, Neuroprosthetics.

\section{INTRODUCTION}

Miniaturization of implantable smart devices for diagnosis and therapeutics is currently compromised by the available means for supplying those devices with electrical energy. Although some ingenious energy harvesting techniques are under research, electrochemical batteries and inductive coupling systems are found in the vast majority of implantable electronic devices; and these two electricity sources comprise bulky parts which, in most cases, are significantly larger than the microelectronic circuitry they feed. One example of his are miniature electrical stimulators for neuroprosthetics.

In 1991, G.E. Loeb and colleagues introduced a novel paradigm for Functional Electrical Stimulation (FES): they conceived and developed smart devices for electrical stimulation of excitable tissues that were small enough for being delivered by injection. These devices, later known by the commercial name BION [1], were intended for solving some major constraints of existing multi-site techniques stimulation. The original, and smallest, BION devices had a length of about $16 \mathrm{~mm}$ and a diameter of $2 \mathrm{~mm}$ and were energized solely by inductive coupling with an external large coil fed by high currents.

However, BION devices fell short of expectations because they were too large and stiff for the originally intended applications. These undesirable features were imposed by the fact that inductive coupling was employed for powering BION devices.

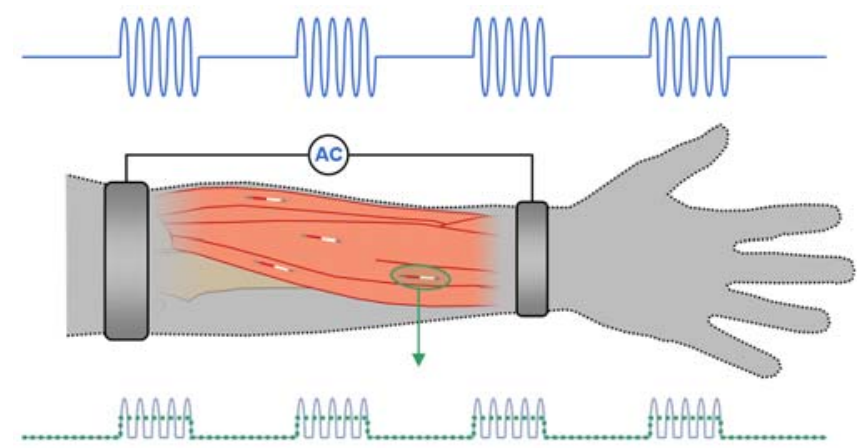

Fig. 1 Illustration of a hypothetical use of the method proposed here for neuroprosthetics. The implants, consisting of a very thin diode and two electrodes in its simplest version, would be implanted by injection within the muscles that require stimulation, close to their motor points. Bursts of $\mathrm{AC}$ current would be forced to flow through tissues by means of external electrodes so that locally, at the vicinity of the capsules, low frequency current densities capable of stimulation would be produced by rectification.

Inductive coupling depends on Faraday's law of induction which states that the induced electromotive force (EMF) in any closed circuit is equal to the time rate of 
change of the magnetic flux through the circuit. Therefore, since the magnetic flux through the circuit is proportional to the area of the surface enclosed by the circuit, then the total area of the circuit generating the EMF, that is, the coil of the implant, is a fundamental parameter for achieving effective inductive coupling between the external magnetic field generator and the implant. BION devices partially overcome this by using a miniature coil with multiple turns and a ferrite core. However, even with multiple turns and a ferrite core, a minimum area (i.e. diameter) is still required for the coil. This is the fundamental reason why BION devices were not made smaller and why it is difficult to conceive devices based on inductive coupling with diameters significantly below $1 \mathrm{~mm} \mathrm{[2].}$

In [3] we proposed and demonstrated a method for performing electrical stimulation with electronic implants: the implanted devices operate as rectifiers of innocuous high frequency $\mathrm{AC}$ currents supplied to the tissue of interest by remote electrodes. In this way, DC or low frequency currents are generated locally around the implants and these resultant currents perform stimulation of excitable tissues. This concept is schematically illustrated in figure 1 .

A key phenomenon for the feasibility of the proposed method is that $\mathrm{AC}$ currents with frequencies above $10 \mathrm{kHz}$ hardly cause any sort of stimulation of excitable tissues; their physiological impact is considered to be solely thermal due to the Joule effect. As a matter of fact, AC currents with frequencies above $10 \mathrm{kHz}$ do stimulate excitable tissues but with much higher magnitude excitation thresholds than those of frequencies in the range from $10 \mathrm{~Hz}$ to $100 \mathrm{~Hz}$; it has been measured that current density thresholds for 1 $\mathrm{MHz}$ currents are above 50 times those of $100 \mathrm{~Hz}$ currents. Therefore, in principle, if the implants can generate current densities with a low frequency (LF, 10 to $100 \mathrm{~Hz}$ ) component that has a magnitude significantly above $1 / 50$ of the high frequency (HF) current density applied by the remote electrodes, it will be possible to perform local stimulation without causing any undesired stimulation of neighbor tissues. In [3] we numerically demonstrated that, for stimulation current density thresholds equivalent to those of BION devices, such LF/HF current density ratios can be easily exceeded and that required HF current densities will not cause thermal damage. Moreover, experimental evidence was provided by an in vivo proof of concept model consisting of an anesthetized earthworm in which a commercial diode was implanted though an incision.

The present paper reports further progress in this technology. We first describe construction and functional test of an injectable stimulator consisting of a single miniature diode $(300 \mu \mathrm{m} \times 300 \mu \mathrm{m} \times 600 \mu \mathrm{m})$ and two thin electrodes which is implanted trough a $19 \mathrm{G}$ needle into an anesthetized earthworm. We then propose a circuit architecture for im- plementing smart implants based on this technology. Both accomplishments are steps towards the implementation of injectable smart microsystems for neuroprosthetics.

\section{INJECTABLE STIMULATOR CONSISTING OF A SINGLE DIODE}

\section{A. Implementation of the implant}

A device consisting of a Schottky diode and two electrodes (Figure 2a) was implemented for implantation in an anesthetized earthworm. The Schottky diode (RB521ZS-30 by Rohm Co., Ltd.) has a length of $0.6 \mathrm{~mm}$, a width of 300 $\mu \mathrm{m}$ and a thickness of $300 \mu \mathrm{m}$. Thin insulated copper wires with a diameter of $150 \mu \mathrm{m}$ (RRP-P-105 by Pro Power) were soldered to the cathode and anode of the diode so that a thin device with a length of about $24 \mathrm{~mm}$ was obtained. The distal $2 \mathrm{~mm}$ of each wire were exposed to form the electrodes of the device. One of the electrodes was bent inwards so that it operated as a hook for implantation with needle (Figure 2b,c). The diode and the solder joints were insulated with a varnish (MR8008B by Electrolube).

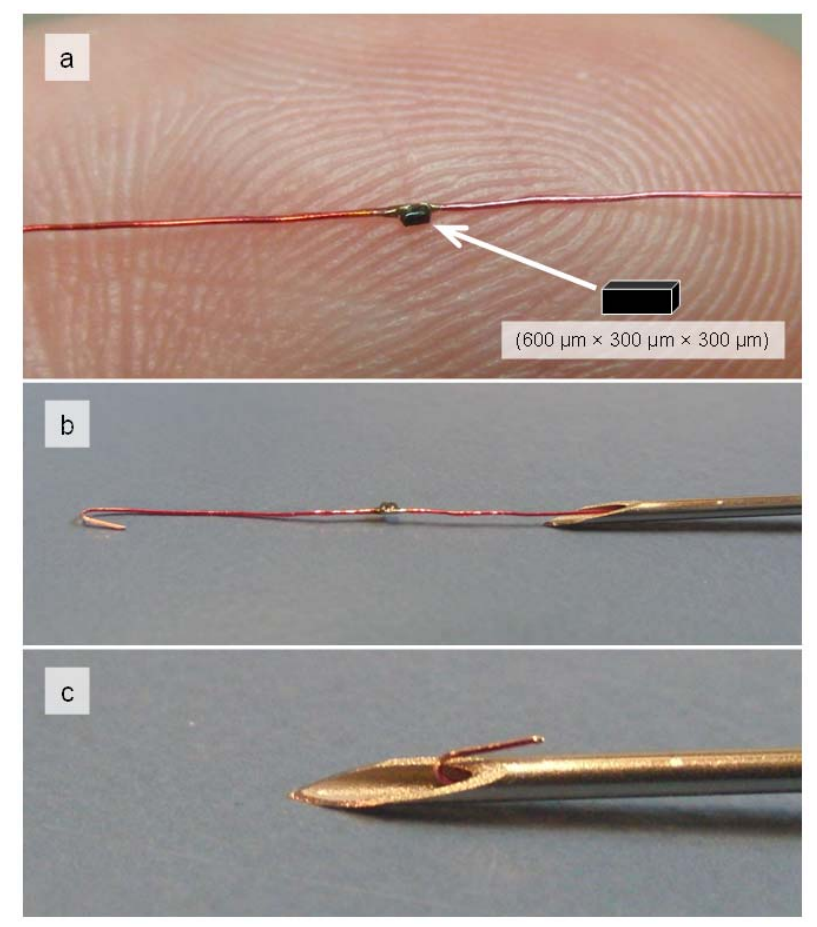

Fig. 2 Pictures of the implemented implant; two wires acting as electrodes soldered to a diode. One of the electrodes is bent to form a hook

\section{B. In vivo test and result}

Live earthworms (Lumbricus sp.) were acquired and kept in a fridge at $10{ }^{\circ} \mathrm{C}$ for a few days before the experiment. Prior to diode implantation, the earthworm was anesthetized 
by immersing it for 15 minutes in a $0.2 \%$ tap water solution of Chlorobutanol (1,1,1-Trichloro-2-Methyl-2-Methyl-2Propanol) (Catalogue number 112054-50G by SigmaAldrich Co., St. Louis, MO, USA).

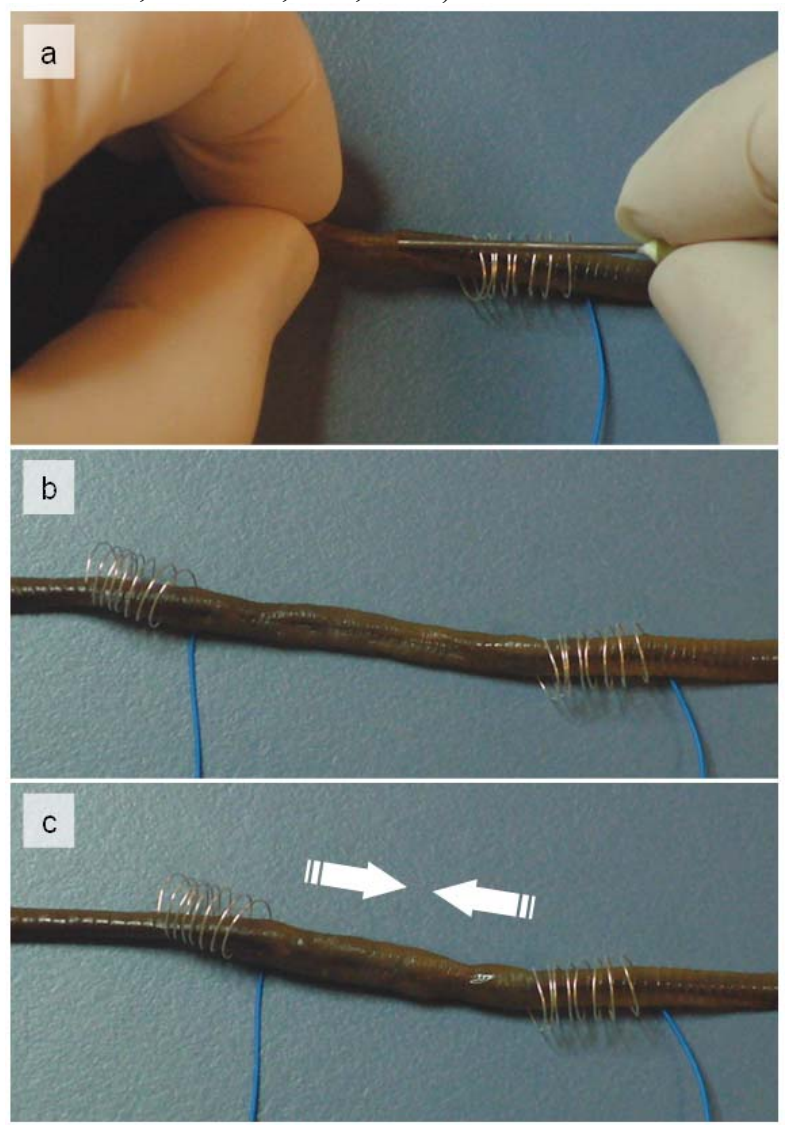

Fig. 3 Pictures of device implantation in an anesthetized earthworm and use. Note that coiled wires are simple electrodes, not inductive coils (see text).

Before device implantation, stimulation was attempted with HF currents. A function generator (model AFG3022 by Tektronix, Inc.) and a custom developed power amplifier were employed for delivering bursts of $100 \mathrm{kHz}$ with amplitude 25 volts, period $10 \mathrm{~ms}$ and duty cycle $50 \%$. Those bursts were applied between two electrodes made of coiled wire at a distance of about $5 \mathrm{~cm}$ (figure $3 \mathrm{~b}$ ), resulting in an electric field magnitude in the order of $5 \mathrm{~V} / \mathrm{cm}$ in the region flanked by the electrodes. As expected, neither contractions nor movements were noticeable.

Then, device was implanted beneath the earthworm dorsal skin by means of a $19 \mathrm{G}$ needle (Fig 2c) as shown in figure $3 \mathrm{a}$. Under these conditions, when the above referred HF bursts were applied, the earthworm contracted very significantly and rapidly in the region where the implant was located (Fig 3c), which demonstrates that the implant was performing electrical stimulation.

\section{Proposed ArChitecture for SMart Stimulators}

As later discussed, the implant configuration used here may allow some simple applications. However, more advanced electronic features will be required for most clinical applications. In particular it will be necessary to control the amount of LF current flowing though the implant and, in addition, it will be required to extract DC current from the HF currents so that control circuitry and other processing circuitry can be powered (e.g. for decoding addresses transmitted with the HF bursts).

Here we propose an integrated circuit architecture that meets both requirements (figure 4). For supplying the implant circuitry, a full wave rectifier with a voltage regulator with small load capacitor is proposed. For controlling the magnitude of LF currents flowing through the implant (i.e. stimulation currents), we propose DAC driven current generators in series with high voltage transistor switches. By activating the left branch transistor, LF current will flow from electrode 2 to 1 whereas, by activating the right branch transistor, LF current will flow from 2 to 1 . This mechanism will enable biphasic stimulation for charge balance.

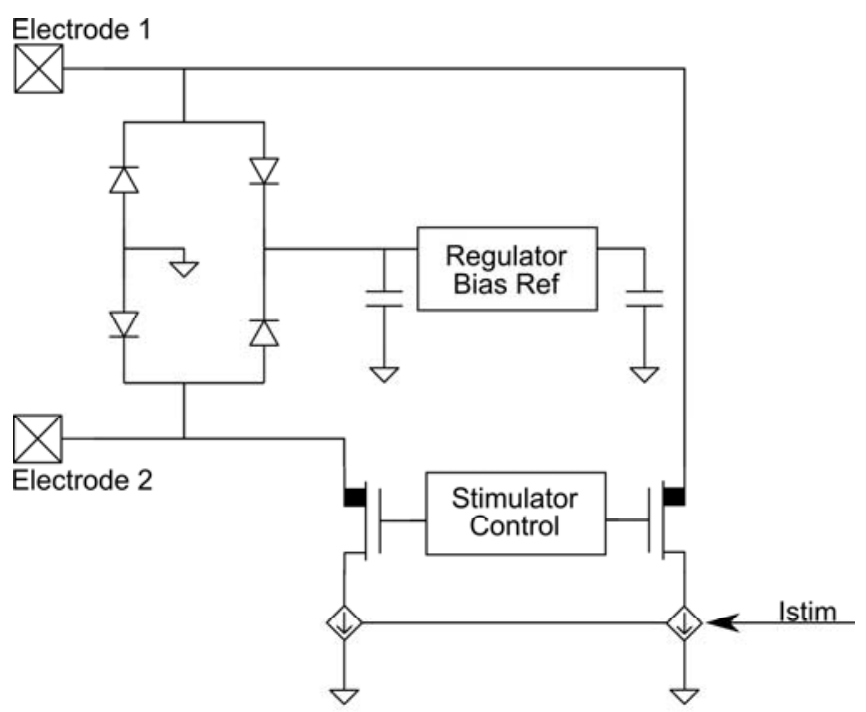

Fig. 4 Proposed architecture for controlling stimulation currents.

\section{Discussion}

As it was previously demonstrated with a larger diode [3], here it has been shown in vivo that an injectable device consisting of a miniature diode and two wires acting as electrodes indeed can generate virtual current pulses able to stimulate muscles when a train of high frequency bursts of current (per se incapable of stimulation) is applied in the region where the implant is located. The simple implant 
configuration used here will allow applications such as temporary or experimental FES systems and therapeutic techniques in which DC currents are required such as local electrochemical ablation of tissues, wound healing and bone fracture repair.

Nevertheless, for other applications it will be required to embed more sophisticated electronics within the implant. In particular, in order to minimize electrochemical damage to the electrodes and tissues in FES clinical applications or in electrical stimulation for pain relief, it will be convenient to include a mechanism for switching the polarity of the rectifier so that charge balance can be achieved. In addition to that, another feature that will be required in neuroprosthetics is current magnitude control. These two features will be achieved by the proposed architecture in section III.

Other electronic functions that will be very interesting to embed within the module are: demodulation of code signals contained within the AC bursts for addressing specific implants and load modulation for transmitting data towards the external systems. With these advanced features embedded within the implant (addressing and data transmission) it is easy to conceive interesting applications of the method such as multi-channel neuroprosthetics systems, recording of action potentials or monitoring of physiological parameters measurable with sensors.

Furthermore, in addition to medical applications, it is possible to envisage other uses of the proposed method such as implantable identification implants. Besides miniaturization, these implants based on the present method may offer some advantages in terms of security over current implantable identification capsules based on inductive or near-field coupling [3].

Currently, complete systems as complex as RFID tags are embedded in integrated circuits with an area of only 0.4 $\mathrm{mm} \times 0.4 \mathrm{~mm}$. Therefore, based on the proposed method, smart stimulation capsules with a diameter below $0.2 \mathrm{~mm}$ should be feasible with existing microelectronic techniques. Moreover, it has to be taken into account that the two ends of the implant can consist of very thin $(<100 \mu \mathrm{m})$ flexible wires. Hence the implants based on the present method may look like short pieces of flexible thread rather than like rigid capsules, as it was the case of previous miniature electrical stimulation implants, such as BION devices.

A drawback of the proposed method is its extremely poor energy efficiency. In a simulated case in [3], it was noted that efficiency was below $0.002 \%$. Nevertheless, such poor energy efficiency does not necessarily rule out portable equipment. As stated in [3], there are practical cases in which the required external energy will be in the order of 1 $\mathrm{W}$, which can be supplied for many hours with batteries.

Somehow in relation with the above it must be noted that we have recently discovered that the proposed concept is not as innovative as claimed in [3]. In 1962, John C. Schuder and colleagues anticipated the idea of using silicon diodes implanted in regions where pulsed RF fields were applied for achieving electrical stimulation. During the 60s and 70 s, they produced a few papers related to this concept [4-6]. However, probably because they were focused on cardiac pacing applications - which demand high current but do not require extreme miniaturization - and mostly because they realized about the poor energy efficiency of the method in comparison to inductive coupling, it seems they abandoned their interest in the method and nobody pursued it any further. Unsurprisingly, they failed to grasp the full potential of the method for neuroprosthetics as at the time microelectronics was in its infancy.

\section{AcKNOWLEDGMENT}

AI's research is supported by a Ramón y Cajal fellowship from the Spanish Ministry for Science and Innovation and a Marie Curie IRG from the European Commission

\section{REFERENCES}

1. Kane MJ, Breen PP, Quondamatteo F, OLaighin G (2011) BION microstimulators: A case study in the engineering of an electronic implantable medical device. Med Eng Phys 33(1): 7-16.

2. Heetderks WJ. (1988) RF powering of millimeter- and submillimetersized neural prosthetic implants. IEEE Trans Biomed Eng 35(5): 323327.

3. Ivorra A (2011) Remote Electrical Stimulation by Means of Implanted Rectifiers. PLoS ONE 6(8): e23456.

4. Schuder JC, Stoeckle H (1964) The silicon diode as a receiver for electrical stimulation of body organs. Trans Am Soc Artif Intern Organs 10:366-70.

5. Mackenzie JW, Schuder JC, Stephenson HE Jr (1967) Radiofrequency energy transport into the body. J Surg Res 7(3):133-44.

6. Schuder JC, Gold JH (1974) Localized DC field produced by diode implanted in isotropic homogeneous medium and exposed to uniform RF field. IEEE Trans Biomed Eng. 21(2):152-63.

\footnotetext{
Author: Antoni Ivorra

Institute: Univeristat Pompeu Fabra

Street: Carrer Roc Boronat 138

City: Barcelona E-081018

Country: Spain

Email: antoni.ivorra@gmail.com
} 